\title{
Computational Modeling of Collicular Integration of Perceptual Responses and Attention in Microsaccades
}

\author{
Ralf Engbert \\ Experimental and Biological Psychology, University of Potsdam, 14476 Potsdam, Germany
}

During visual fixation on a target object, our eyes are not motionless but generate slow fixational eye movements and microsaccades. Effects of visual attention have been observed in both microsaccade rates and spatial directions. Experimental results, however, range from early $(<200 \mathrm{~ms})$ to late $(>600 \mathrm{~ms})$ effects combined with cue-congruent as well as cue-incongruent microsaccade directions. On the basis of well characterized neural circuitry in superior colliculus, we construct a dynamical model of neural activation that is modulated by perceptual input and visual attention. Our results show that additive integration of low-level perceptual responses and visual attention can explain microsaccade rate and direction effects across a range of visual cueing tasks. These findings suggest that the patterns of microsaccade direction observed in experiments are compatible with a single dynamical mechanism. The basic principles of the model are highly relevant to the general problem of integration of low-level perception and top-down selective attention.

\section{Introduction}

Microsaccades are rapid, small-amplitude eye movements that are generated involuntarily during visual fixation (Barlow, 1952; Zuber et al., 1965; Steinman et al., 1973). The function of microsaccades for vision has been investigated extensively (MartinezConde et al., 2006; Rucci et al., 2007; Hafed, 2011; for review, see Martinez-Conde et al., 2004, 2009). For example, recent behavioral findings suggest that microsaccades provide a mechanism for precisely relocating gaze in high-acuity tasks (Ko et al., 2010). The primate superior colliculus (SC), the top-level brainstem structure involved in the control of saccadic eye movements (Carello and Krauzlis, 2004; McPeek and Keller, 2004), has been identified as the key neural structure for microsaccade generation (Hafed et al., 2009; Hafed and Krauzlis, 2012).

Since statistical properties of microsaccades are sensitive to changes of perceptual input as well as to modulations of cognitive states (Hafed and Clark, 2002; Engbert and Kliegl, 2003), the analysis of fixational eye movements and microsaccades might provide new insights into the integration of low-level perceptual signals and top-down selective attention (Posner, 1980). In a typical attentional cueing task, effects of covert attention on microsaccades did not depend on the subsequent response type (e.g., saccade or key press; Engbert and Kliegl, 2003). As a first effect of visual or auditory stimulus onsets, a microsaccade rate modulation is observed experimentally (Fig. $1 A$ ). A decrease in microsaccade rate is found during a time window from $\sim 100$ to $200 \mathrm{~ms}$

\footnotetext{
Received Feb. 20, 2012; revised April 20, 2012; accepted April 27, 2012.

Author contributions: R.E. designed research; R.E. performed research; R.E. analyzed data; R.E. wrote the paper.

This research was supported by Deutsche Forschungsgemeinschaft (Research Unit 868, Grant EN 471/3-2). I thank Jochen Laubrock, Sven Ohl, and Petra Sinn for valuable discussions.

The author declares no competing financial interest.

Correspondence should be addressed to Ralf Engbert, Experimental and Biological Psychology, Cognitive Science Program, University of Potsdam, Karl-Liebknecht-Strasse 24-25, 14476 Potsdam, Germany. E-mail: ralf.engbert@uni-potsdam.de.

DOI:10.1523/JNEUROSC1.0808-12.2012

Copyright $\odot 2012$ the authors $\quad 0270-6474 / 12 / 328035-05 \$ 15.00 / 0$
}

after stimulus onset. After this microsaccadic inhibition effect, an epoch of microsaccadic enhancement starts $\sim 200-250 \mathrm{~ms}$ after the display change and extends up to $500 \mathrm{~ms}$, depending on the details of the experimental setup (see also Pastukhov and Braun, 2010; Hafed et al., 2011; for review, see Engbert, 2006). This pattern of rate modulations is qualitatively similar to saccadic inhibition (Reingold and Stampe, 2002), i.e., the decrease in saccade rate following display changes.

In addition to microsaccadic rate modulations, experiments with covert spatial attention shifts indicated that the distribution of microsaccade directions can be influenced by attentional cues. The first studies on fixational eye movements in the context of attentional cueing tasks (Fig. $1 A$ ) indicated that microsaccades produce a cue-congruent bias (Hafed and Clark, 2002; Engbert and Kliegl, 2003); however, a series of later experiments demonstrated that both cue-congruent and cue-incongruent microsaccadic responses can be found (Rolfs et al., 2004, 2005; Laubrock et al., 2005) (Fig. 1B). Thus, theoretical models of microsaccadic rate and direction effects need to explain a complicated temporal pattern of responses.

\section{Materials and Methods}

Baseline model. We model the generation of fixational eye movements by neural activity in superior colliculus (Hafed et al., 2009) on a finite square lattice of $L \times L$ of continuous activations with periodic boundary conditions. We implement a self-avoiding walk (Freund and Grassberger, 1992) as an explicit model for the generation of neural noise (Engbert et al., 2011). In each time step, activation is added to the current position $(i, j)$ of the walker,

$$
h_{i j} \rightarrow h_{i j}+1
$$

while all other lattice sites $(k, l) \neq(i, j)$ relax slowly according to the equation,

$$
h_{k l} \rightarrow(1-\varepsilon) \cdot h_{k l}
$$


To keep the walker at an intended fixation position, we simulate the self-avoiding walk in a 2D quadratic potential $u(i, j)$ of the form,

$$
\begin{aligned}
u(i, j)=\lambda L(a & (t)\left(\frac{i-i_{0}}{i_{0}}\right)^{2} \\
& \left.+b(t)\left(\frac{j-j_{0}}{j_{0}}\right)^{2}\right),
\end{aligned}
$$

where the parameter $\lambda$ is used to adjust the steepness of the potential and $\left(i_{0}, j_{0}\right)$ represents the rostral pole of the SC related to movements with vanishing movement amplitude. For the baseline model, the time-varying functions $a(t)$ and $b(t)$ are fixed at a constant value, i.e., $a(t)=b(t)=1$. The potential is a parsimonious concept to implement a mechanism to keep the eyes at an intended target position. The walker moves to the lattice position among the four neighboring sites with the smallest value of the sum of selfgenerated activation and potential. We interpret the numerical value of $h_{i j}$ as the neural activation of a local population of SC neurons that drive fixational eye movements (Engbert et al., 2011).

A microsaccade is triggered when the walker reaches a lattice site with activation higher than a predefined critical value, i.e., $h_{i j}>h_{c}$. The target location for a microsaccade is given by the lattice site which represents the global minimum of activation. In humans, a preference for horizontal and vertical microsaccade directions is observed (Engbert and Kliegl, 2003). Such a preference could result from the dissociation into horizontal and vertical saccade centers (Sparks, 2002) with independent activity patterns for fixational eye movements. In the model (Engbert et al., 2011), we introduced an additional oculomotor potential to account for the preferences in microsac-

cade directions. All model parameters were taken from the original publication of the model (Engbert et al., 2011), i.e., $L=51, i_{0}=j_{0}=25, \varepsilon=$ $10^{-3}, \lambda=1$, and $h_{c}=7.9$.

Effects of display changes and covert attention shifts. The modeling strategy in the current work is based on the hypothesis that peripheral stimulus onsets introduce a transient asymmetrical change of the potential. Neurons in the SC representing the peripheral part of the visual field change their activity during covert attention (Fecteau et al., 2004; Ignashchenkova et al., 2004). The transient change of the potential in our model is used as a mathematical tool to introduce a possible coupling mechanism of peripheral SC activity to the activity in the rostral pole that modulates fixational eye movements. A similar approach was chosen by Hafed et al. (2009) in simulations of the activity distribution in SC. Note that the potential studied here is more fundamental than the activity distribution, since the potential drives the activity in our model. More specifically, temporal variations of microsaccade rate and direction were modeled by transient changes of the potential (Fig. $2 B$ ) with time-varying functions $a(t)$ and $b(t)$ and a time-dependent threshold $h_{c}(t)$. First, we model the impact of perceptual input on the slope of the potential by functions:

$a_{p}(t)=b_{p}(t)=\frac{1}{1+C\left(t-\tau_{p}\right)}$

$$
\text { with } C(t)=\lambda_{1} \exp \left(-\rho_{1} t^{2}\right) \text {. }
$$

Second, the attentional variation of the potential is specified as:

$$
\begin{aligned}
& a_{A}(t \geq 0)=\frac{1}{1+D\left(t-\left(\tau_{p}+\tau_{A}\right)\right)} \\
& \quad a_{A}(t<0)=b_{A}(t)=1,
\end{aligned}
$$
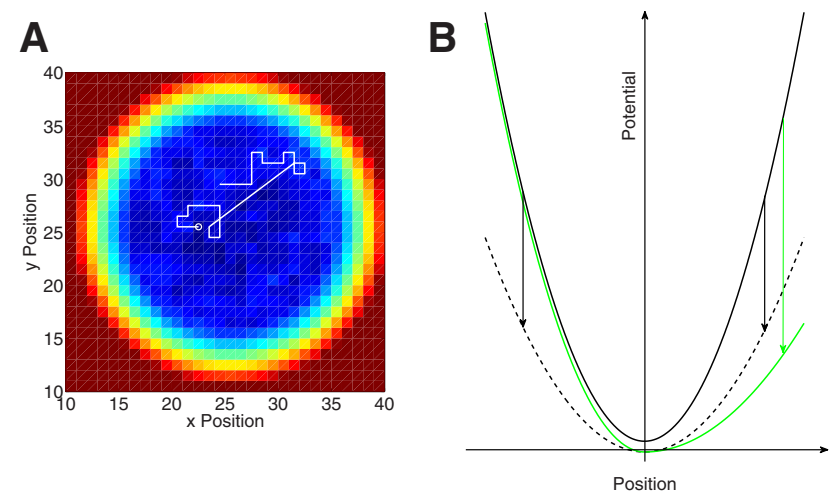

Figure 2. Self-avoiding walk in a potential. $\boldsymbol{A}$, A random walk on a discrete square lattice builds up a self-generated activation at each lattice point. The sum of self-generated activation and an additional movement potential determine movement direction. Visiting a lattice site with overcritical activation induces a microsaccade (straight line) to the global minimum of the sum of activation and potential. $\boldsymbol{B}$, Temporal variations of the movement potential occur as a result of changes in perceptual input and attentional effects. The changes are transient and are different for low-level perceptual effects (dashed line) and directional attention effects (green line).

$$
\text { with } D(t)=\lambda_{2} \frac{\rho_{2}^{\kappa}}{\Gamma(\kappa+1)} t^{\kappa} \exp \left(-\rho_{2} t\right),
$$

where the two free parameters $\kappa$ and $\tau_{\mathrm{A}}$ account for possible changes in waveform (parameter $\kappa$ gives the order of a polynomial for short time scales while the tail of the function $D(t)$ is an exponential decay that is independent of the value of $\kappa$ ) and temporal delay $\tau_{\mathrm{A}}$ with respect to the 
A
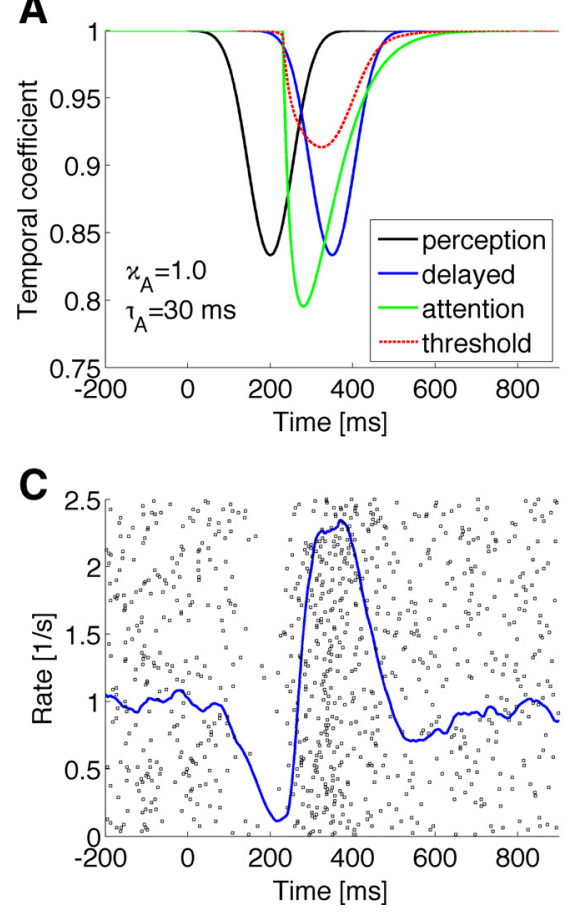

B

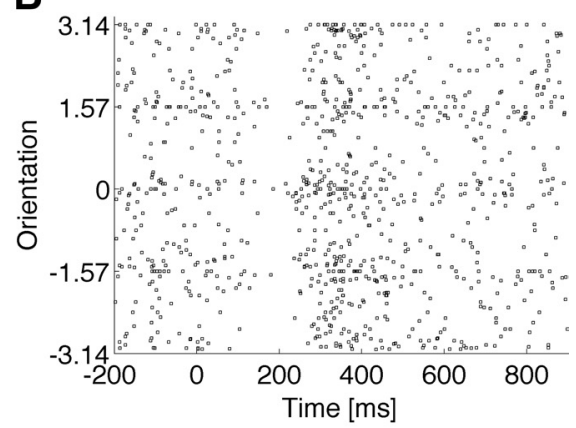

D

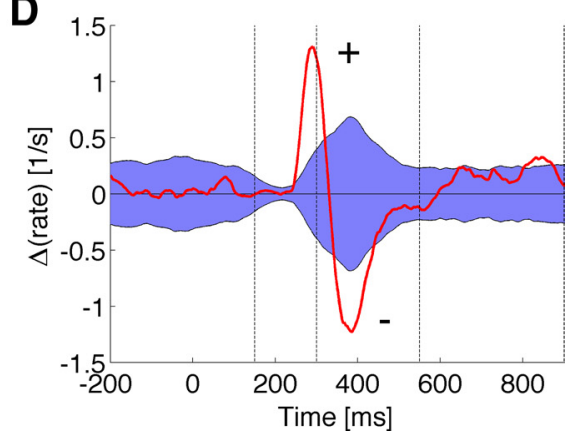

Figure 3. Modulation of microsaccade rate and direction. $A$, Transient variations of the potential due to perceptual input change (black line) and attention (green) and resulting threshold variation (red dashed line). $\boldsymbol{B}$, Microsaccadic directions computed from 2000 runs of the model (raster plot). $\boldsymbol{C}$, Corresponding rate modulation (blue line) computed from raster plot (where each line represents a single model run). $\boldsymbol{D}$, Rate difference between cue-congruent and cue-incongruent microsaccades (red line) and bootstrap-based confidence region around the zero difference.
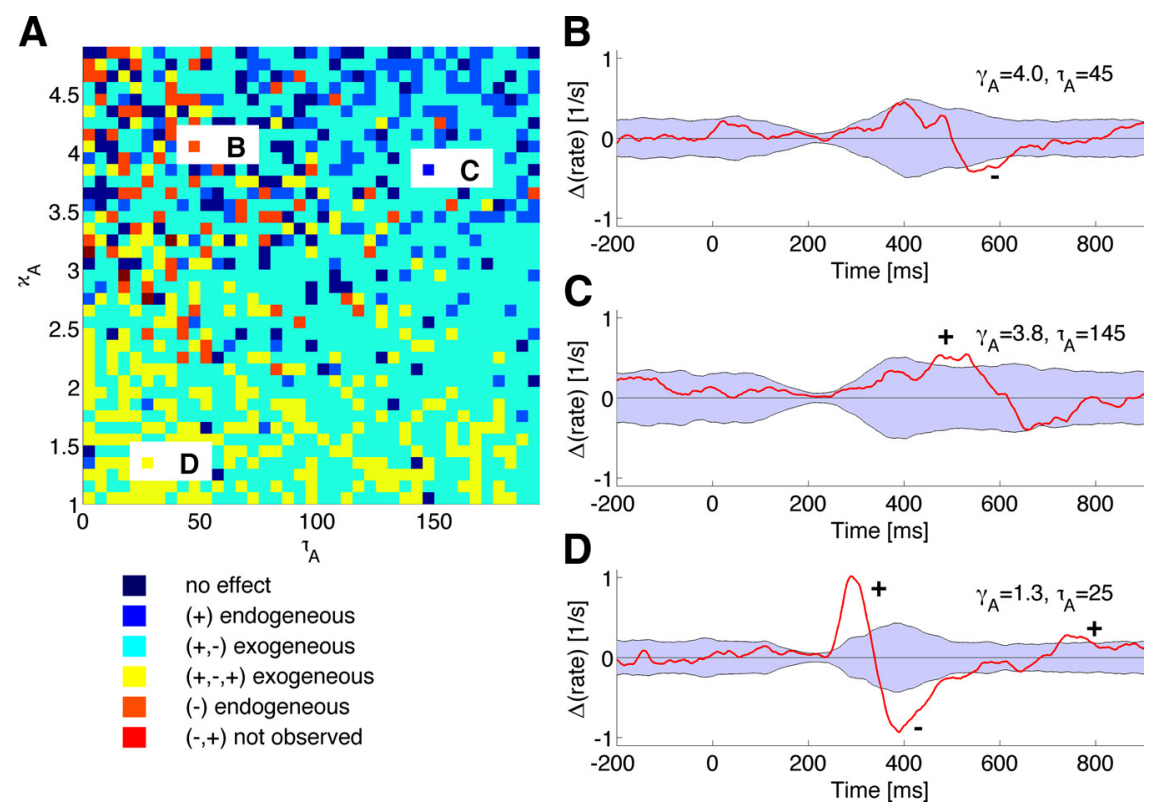

Figure 4. Qualitative analysis of the model's dynamics. $\boldsymbol{A}$, Variations of attentional delay $\tau_{\mathrm{A}}$ and waveform exponent $\kappa_{\mathrm{A}}$ indicates that three regions with endogenous cue-congruent (blue), endogenous cue-incongruent (orange), exogenous (red) pattern of microsaccadic direction effects are reproduced by the model. $\boldsymbol{B}-\boldsymbol{D}$, Patterns of microsaccadic direction effects as a function of time for three different combinations of model parameters highlighted in the $2 \mathrm{D}$ plot in $\boldsymbol{A}$.

perceptual change. Third, the attentional change $a_{\mathrm{A}}(t)$ and a delayed version of perceptual change $f(t)=a_{\mathrm{P}}\left(t-\tau_{\mathrm{P}}\right)$ are additively integrated to result in a function determining the threshold variation for microsaccade triggering, i.e.,

$$
h_{c}(t)=\frac{1}{1+\beta\left((1-f(t))+\left(1-a_{\mathrm{A}}(t)\right)\right)} .
$$

The model parameters were set to $\tau_{\mathrm{P}}=150 \mathrm{~ms}$, $\lambda_{1}=0.2, \lambda_{2}=0.7, \rho_{1}=0.0002, \rho_{2}=0.02$, and $\beta=0.3$.

In Figure $3 A, \kappa=1.0$ and $\tau_{\mathrm{A}}=30 \mathrm{~ms}$. In Figure $4 A$, parameters $\kappa$ and $\tau_{\mathrm{A}}$ were varied systematically. The model's spatial distribution of activation $\left\{h_{i j}\right\}$ is self-generated; earlier simulations indicated that it takes several thousand iterations for a stationary distribution to evolve (Engbert et al., 2011). Therefore, the first 5000 iterations were discarded from each model run to remove transients from the statistical analyses of the model's behavior.

\section{Results}

In any successful computational model, microsaccades should be generated as a consequence of neural noise (Otero-Millan et al., 2011), which is supported by exponentially distributed inter-microsaccade intervals, compatible with a Poisson process of temporally independent events (Engbert, 2006; Mergenthaler and Engbert, 2010). However, recent experiments indicated that microsaccades might also be part of the process of gaze-control in high-acuity tasks (Ko et al., 2010). The kinematic similarity between saccades and microsaccades lends support to a subthreshold mechanism for the generation of microsaccades within the same neural circuitry as for voluntary saccades (Zuber et al., 1965; Otero-Millan et al., 2008; Hafed and Krauzlis, 2012). Furthermore, models should be evaluated based on a number of criteria (Otero-Millan et al., 2011): The distributions of saccadic intervals, the relation between microsaccades and slow movements (drift), and the timing and waveforms of microsaccadic inhibition.

We used a recently proposed integrated model of fixational eye movements and microsaccades (Engbert et al., 2011) to simulate microsaccades under the influence of display changes and selective attention. The model reproduced a range of behavioral findings based on an emergent dynamical coupling of microsaccades and slow movements (Engbert and Kliegl, 2004; Engbert and Mergenthaler, 2006) and might provide an adequate theory for the statistical properties of slow fixational eye movements and microsaccades. The dynamic principle underlying the model is a self-avoiding random walk (Freund and Grassberger, 1992) in a potential as an integrative framework for the generation of both slow movements (physiological drift) and microsaccades (Engbert et al., 2011), i.e., drift (slow movements) and microsaccades were produced by the same laws of motion.

In the model, activation is increased at the walker's current position while all other lattice sites relax (Fig. $2 \mathrm{~A}$ ). A movement potential, which is added to the self-generated activation field, keeps the walker at an intended fixation position (Fig. $2 B$ ). The 
walker tends to a local minimum of the sum of self-generated activation and potential (slow movements). A microsaccade to the global minimum across the lattice is generated, when the walker visits a lattice site with overcritical activation (see Materials and Methods).

Since the model's movement potential is the dynamical mechanism underlying the intention to fixate a particular location, a temporal variation of the slope of the potential can be used to implement effects of display changes and top-down selective attention (Hafed and Clark, 2002; Engbert and Kliegl, 2003; Rolfs et al., 2004, 2005; Laubrock et al., 2005). We assume that a display change induces a transient global reduction of the slope of the potential (Fig. 2B, dashed line), while an attentional shift to the periphery introduces an asymmetrical deformation of the potential toward the locus of attention (Fig. $2 B$, green line). This assumption is compatible with earlier simulations of changes of neural activity in microsaccade generation (Hafed et al., 2009).

Our model simulations are based on three critical assumptions (see Materials and Methods). First, we assume that the transient reduction of the potential (Fig. $3 A$, black line) by changes in perceptual input is followed by a delayed signal that is forwarded to the trigger for microsaccades (Fig. 3A, blue line). Second, we postulate that attention causes a similar, but asymmetrical change of the potential that follows the display change with a variable temporal delay and a specific waveform determined by the properties of the attentional cue (Fig. $3 A$, green line). Third, both signals are integrated additively and result in a threshold variation that is determined by both perceptual input and attentional response (Fig. $3 A$, dotted red line). Such an integration mechanism is compatible with the neuroanatomical structure of the SC with well characterized sensory and motor layers and a functional connection (Lee et al., 1997).

The immediate response of the model's intrinsic dynamics to the transient change of the potential is a reduction of the microsaccade rate (Fig. $3 C$ ), while a later change of the threshold generates the overshoot in microsaccade rate observed in experiments. It is important to note that this change of the potential does not introduce a direction bias, which is induced only later by effects of covert attention (Fig. 3B,D). When rate differences of cue-congruent and cueincongruent microsaccades are plotted (Fig. $3 D$ ), an early and strong attentional variation of the potential generates a pattern of immediate cue-congruent, an intermediate cue-incongruent, and a late cuecongruent microsaccadic direction bias. For statistical evaluation of the obtained direction patterns, we applied a rate-estimation procedure with a causal window developed for spike train analysis (Dayan and Abbott, 2001; see also Engbert, 2006). Randomization of microsaccade directions was used to compute confidence regions around the baseline of a zero difference in microsaccade directions (Fig. 3D, blue area). The resulting statistically significant patterns of directions are typically observed in experiments with exogenous flash cues (Laubrock et al., 2005).

Based on systematic numerical simulations of the model, we identified the two critical parameters of the attentional cues that had a strong impact on the interaction of both rate and directions of microsaccades. First, the delay of the earliest effect of selective attention relative to the immediate perceptual effect on the movement potential varies between exogenous flash-type visual cues or auditory cues (short delay) and endogenous symbolic cues that need top-down processing before an attentional response can be generated across the oculomotor pathway (long delay). Second, the waveform of the attentional response can in principle range from a steep and short ( $\kappa$ small) to a slow and more sustained ( $\kappa$ large) transient reduction of the potential. A systematic variation of both parameters indicates that the model predicts the typical effects of attention on microsaccades found in experiments (Fig. 4A). Auditory cues produce intermediate cueincongruent effects (Rolfs et al., 2005) that are captured by model simulations with a short-delay, but slow potential change (Fig. $4 B)$. Simulation of endogenous cues which produce a cuecongruent microsaccade bias (Hafed and Clark, 2002; Engbert and Kliegl, 2003) are obtained for longer delays (Fig. 4C). Finally, the model generated the more complicated pattern with cue-congruent as well as cue-incongruent microsaccade effects for exogenous cues (Laubrock et al., 2005; Rolfs et al., 2005) (Fig. 4D).

\section{Discussion}

Microsaccades recorded during visual fixation before a motor response to a peripheral stimulus provide an important tool for the analysis of vision, attention, and eye movements. The present study proposed a mathematical framework to simulate influences of peripheral stimulus onsets on microsaccades during visual fixation. We identified two critical parameters of experimental manipulations of attention: (1) the temporal delays of the attention effects relative to stimulus onset and (2) the waveform (or steepness) of the attentional onset. Variations of these two parameters could explain complicated patterns of attentional effects on microsaccades observed in experiments. The simulation of the model suggest that properties of the attentional manipulations interact with the intrinsic dynamics of fixational eye movements (i.e., slow movements, microsaccades, and their interaction) to reproduce the observed patterns of microsaccade rate and direction effects.

In our model, low-level perceptual responses and top-down attentional modulation are integrated to produce the highly specific responses of microsaccades across a number of attentional cueing tasks. While the low-level responses are strongly expressed in microsaccade rates, the effects of selective attention are observed in microsaccade directions. The model proposed and analyzed in this study is based on the assumption of an additive integration of both processes as a single mechanism to explain a range of experimentally observed effects. These results extend earlier simulations of neural activation underlying the control of microsaccades in superior colliculus (Hafed et al., 2009; Hafed and Krauzlis, 2012).

Our model might be extended to the more general problem of saccadic inhibition (Reingold and Stampe, 2002; Buonocore and McIntosh, 2008), where a transient change of a scene display during a visual task induces a decrease in saccade rate. Solutions to the problem of integration of low-level perceptual signals (Martinez-Conde et al., 2000; Rucci and Casile, 2004; Hafed and Krauzlis, 2010) and top-down attention (Posner, 1980; Hafed et al., 2011) studied here in the context of microsaccades might contribute to other aspects of this long-standing issue in active vision (Findlay and Walker, 1999) and scene perception (Itti and Koch, 2001).

\section{References}

Barlow HB (1952) Eye movements during fixation. J Physiol 116:290-306. Buonocore A, McIntosh RD (2008) Saccadic inhibition underlies the remote distractor effect. Exp Brain Res 191:117-122.

Carello CD, Krauzlis RJ (2004) Manipulating intent: evidence for a causal role of the superior colliculus in target selection. Neuron 43:575-583.

Dayan P, Abbott LF (2001) Theoretical neuroscience. Computational and mathematical modeling of neural systems. Cambridge, MA: MIT.

Engbert R (2006) Microsaccades: a microcosm for research on oculomotor control, attention, and visual perception. Prog Brain Res 154:177-192. 
Engbert R, Kliegl R (2003) Microsaccades uncover the orientation of covert attention. Vision Res 43:1035-1045.

Engbert R, Kliegl R (2004) Microsaccades keep the eyes' balance during fixation. Psychol Sci 15:431-436.

Engbert R, Mergenthaler K (2006) Microsaccades are triggered by low retinal image slip. Proc Natl Acad Sci U S A 103:7192-7197.

Engbert R, Mergenthaler K, Sinn P, Pikovsky A (2011) An integrated model of fixational eye movements and microsaccades. Proc Natl Acad Sci U S A 108:E765-E770.

Fecteau JH, Bell AH, Munoz DP (2004) Neural correlates of the automatic and goal-driven biases in orienting spatial attention. J Neurophysiol 92:1728-1737.

Findlay JM, Walker R (1999) A model of saccade generation based on parallel processing and competitive inhibition. Behav Brain Sci 22:661-674.

Freund H, Grassberger P (1992) The red queen's walk. Physica A 190:218-237.

Hafed ZM (2011) Mechanisms for generating and compensating for the smallest possible saccades. Eur J Neurosci 33:2101-2113.

Hafed ZM, Clark JJ (2002) Microsaccades as an overt measure of covert attention shifts. Vision Res 42:2533-2545.

Hafed ZM, Krauzlis RJ (2010) Microsaccadic suppression of visual bursts in the primate superior colliculus. J Neurosci 30:9542-9547.

Hafed ZM, Krauzlis RJ (2012) Similarity of superior colliculus involvement in microsaccade and saccade generation. J Neurophysiol 107:1904-1916.

Hafed ZM, Goffart L, Krauzlis RJ (2009) A neural mechanism for microsaccade generation in the primate superior colliculus. Science 323:940-943.

Hafed ZM, Lovejoy LP, Krauzlis RJ (2011) Modulation of microsaccades in monkey during a covert visual attention task. J Neurosci 31:15219-15230.

Ignashchenkova A, Dicke PW, Haarmeier T, Thier P (2004) Neuronspecific contribution of the superior colliculus to overt and covert shifts of attention. Nat Neurosci 7:56-64.

Itti L, Koch C (2001) Computation modeling of visual attention. Nat Rev Neurosci 2:194-203.

Ko HK, Poletti M, Rucci M (2010) Microsaccades precisely relocate gaze in a high visual acuity task. Nat Neurosci 13:1549-1553.

Laubrock J, Engbert R, Kliegl R (2005) Microsaccade dynamics during covert attention. Vision Res 45:721-730.

Lee PH, Helms MC, Augustine GJ, Hall WC (1997) Role of intrinsic synaptic circuitry in collicular sensorimotor integration. Proc Natl Acad Sci U S A 94:13299-13304.

Martinez-Conde S, Macknik SL, Hubel DH (2000) Microsaccadic eye movements and firing of single cells in the striate cortex of macaque monkeys. Nat Neurosci 3:251-258.
Martinez-Conde S, Macknik SL, Hubel DH (2004) The role of fixational eye movements in visual perception. Nat Rev Neurosci 5:229-240.

Martinez-Conde S, Macknik SL, Troncoso XG, Dyar TA (2006) Microsaccades counteract fading during fixation. Neuron 49:297-305.

Martinez-Conde S, Macknik SL, Troncoso XG, Hubel DH (2009) Microsaccades: a neurophysiological analysis. Trends Neurosci 32:463-475.

McPeek RM, Keller EL (2004) Deficits in saccade target selection after inactivation of superior colliculus. Nat Neurosci 7:757-763.

Mergenthaler K, Engbert R (2010) Microsaccades are different from saccades in scene perception. Exp Brain Res 203:753-757.

Otero-Millan J, Troncoso XG, Macknik SL, Serrano-Pedraza I, MartinezConde S (2008) Saccades and microsaccades during visual fixation, exploration, and search: foundations for a common saccadic generator. J Vis 8:21.1-21.18.

Otero-Millan J, Macknik SL, Serra A, Leigh RJ, Martinez-Conde S (2011) Triggering mechanisms in microsaccade and saccade generation: a novel proposal. Ann NY Acad Sci 1233:107-116.

Pastukhov A, Braun J (2010) Rare but precious: Microsaccades are highly informative about attentional allocation. Vision Res 50:1173-1184.

Posner MI (1980) Orientation of attention. The VIIth Sir Frederic Bartlett lecture. Q J Exp Psychol 32:3-25.

Reingold EM, Stampe DM (2002) Saccadic inhibition in voluntary and reflexive saccades. J Cogn Neurosci 14:371-388.

Rolfs M, Engbert R, Kliegl R (2004) Microsaccade orientation supports attentional enhancement opposite to a peripheral cue. Psychol Sci 15:705-707.

Rolfs M, Engbert R, Kliegl R (2005) Crossmodal coupling of oculomotor control and spatial attention in vision and audition. Exp Brain Res 166:427-439.

Rucci M, Casile A (2004) Decorrelation of neural activity during fixational instability: possible implications for the refinement of V1 receptive fields. Vis Neurosci 21:725-738.

Rucci M, Iovin R, Poletti M, Santini F (2007) Miniature eye movements enhance fine spatial detail. Nature 447:851-854.

Sparks DL (2002) The brainstem control of saccadic eye movements. Nat Rev Neurosci 3:952-964.

Steinman RM, Haddad GM, Skavenski AA, Wyman D (1973) Miniature eye movement. Science 181:810-819.

Zuber BL, Stark L, Cook G (1965) Microsaccades and the velocityamplitude relationship for saccadic eye movements. Science 150:14591460 